\title{
OSPF vs EIGRP: A Comparative Analysis of CPU Utilization using OPNET
}

\author{
Muhammad Kashif Hanif*, Ramzan Talib ${ }^{\dagger}$, Nafees Ayub $^{\ddagger}$, Muhammad Umer Sarwar ${ }^{\S}$, and Sami Ullah ${ }^{\llbracket}$ \\ Department of Computer Science, \\ Government College University, Faisalabad, Pakistan
}

\begin{abstract}
Routing is difficult in enterprise networks because a packet might have to traverse many intermediary nodes to reach the final destination. The selection of an appropriate routing protocol for a large network is difficult task. The focus of this work is to select and identify the best routing technique for a computer network. In this study, the performance of OSPF and EIGRP routing protocols with respect to $\mathrm{CPU}$ utilization is analyzed using OPNET simulator. The results depict EIGRP acquires redundant information which effect CPU utilization.
\end{abstract}

Keywords-Network protocols; topology; OPNET; interior gateway protocols (IGPS); OSPF;

\section{INTRODUCTION}

In this present era, computer networks are growing rapidly day by day. Communication technologies provide user convenient services such as file sharing/transferring, printer sharing, video streaming and video/voice conferencing. Internet is the global network of interconnected computers. Internet plays an important role in todays communication networks which are based on technology that provides the technical infrastructure. In this technical infrastructure routing protocols are used to find an efficient route to transmit packets across the internet.

In WAN, IP packets are being forwarded by the routers. For this purpose the routing devices use the routing protocols which determine and then select the best path to forward the packets [1]. Communication among different routing protocols depends on routing algorithms which base on the nodes to determine the route to forward the packet over networks [2]. Routing in internet plays an important role so it is the heartbeat of the internet. Routing protocols are comprised into two diverse categories: Interior gateway protocols (IGPs) and Exterior gateway protocols (EGPs). The IGPs such as OSPF and EIGRP work in an autonomous system routing whereas the EGPs (such as BGP) work for routing among multiple autonomous systems. Our effort in this research is to investigate the IGPs so the purpose of this investigation is to examine the OSPF and EIGRP regarding traffic-load and CPU utilization by nodes.

As the use of internet growing day by day, ISPs are trying to encounter the traffic demands with new technologies and enhanced existing resources. Network utilization depends on routing of data packets on a network because a packet follows a path to reach its destination. Intra-domain internet routing protocol mostly uses the OSPF to determine the best path for packets [3].
OSPF uses a topology to determine the short path. For this purpose it produces the link-state packets from each router in a network and these packets contain the updated information for routers. So OSPF uses this information to determine the path. If any change occurs in a network then recalculation process occurs [4].

Extended IGRP is an improved form of IGRP (interior gateway routing protocol). This improvement is the resultant of changes in routing and changes in demands of internet works. In this improvement, abilities of link-state protocols have been integrated into distance-vector protocols. Extended IGRP also consists of some vital protocols that increase its working competence than other different routing protocols. It uses DUAL that enables it to find whether the advertised path by a neighbor router is looped or loop-free. DUAL also permits the EIGRP to determine another route without waiting for updated routing information from other routers [5].

The objective of this work is to find routing protocol which has better CPU utilization and enhanced performance. Network administrators can use this study to select a protocol for computer network. In this work, protocols are analyzed with respect to the CPU utilization and link-state advertisements by considering various scenarios. The reminder of the paper is organized in different sections. Section II discusses related work. Section III describes the research methodology. The results are presented in Section IV. Section V concludes the outcomes.

\section{RELATED WORK}

Agarwal et al. examined the effect of CPU utilization of Border Gateway Protocol (BGP) from multiple routers in the Sprint IP network. They correlated BGP with SNMP data to measure the CPU Utilization of 200 routers. The results showed BGP uses the majority of CPU cycles for short time slice. This is due to increased size of BGP routing table [6]. Shah and Rana analyzed the convergence and traffic for RIP and OSPF within network using OPNET simulator. Convergence time of OSPF single area is greater than OSPF multi area and OSPF multi stub area [7]. Nazumudeen and Mahendran compared the OSPF, EIGRP, RIPv1 and RIPv2 routing protocols to determine which protocol is suitable for a network. EIGRP performs better than RIP and OSPF due to speedy convergence process, great handling, and improved scalability of routing loops [8].

Kudtarkar et al. compared IGPs protocols using WFQ 
technique by establishing dissimilar scenarios in OPNET. EIGRP perform better for non-real time applications while OSPF and IGRP have better performance for real time applications [9]. Vetriselvan et al. analyzed IGRP, EIGRP, RIP, and OSPF protocols to evaluate the performance for slip8_gateway routers. Simulations showed OSPF has minimum transmission cost and IGRP has maximum overhead. Moreover, OSPF and EIGRP has better for maximum throughput and link utilization, respectively [10].

Patel and Pandey discussed the necessity to advertise route among routers for multiple routing protocols and autonomous systems in hybrid network. Moreover, they analyzed the the route summarization and redistribution for OSPF and EIGRP protocols. The route distribution and summarization reduces the memory, CPU utilization, and network traffic [11]. Masood et al. compared OSPF, EIGRP, and RIP using NSFnet topology over 14 nodes. OSPF showed better convergence while RIP has low CPU Utilization [12]. Stankoska et al. studied OSPF and EIGRP with respect to the convergence time, end-to-end delay, packet loss, jitter and throughput for video streaming and voice conferencing. EIGRP achieved better convergence, throughput and less packet loss. Researchers have not evaluated the routing protocols for spoke-to-hub topology. In this work, we study the OSPF and EIGRP for spoke-to-hub topology [13].

\section{Methodology}

Different network scenarios were designed for spoke-tohub topology to analyze the impact of CPU utilization for OSPF and EIGRP protocols. OPNET simulator was used to design the network scenarios and investigate the performance of these protocols over the designed scenarios. Spoke-to-hub network topology was designed using Cisco 2600, 7500 routers and switches. Within this topology, Local Area Networks (LAN), 100 Base T Ethernet link and Point to Point T1 link are used. The single area OSPF is used for this study.

Fig. 1 and 2 shows the scenarios where the EIGRP and OSPF protocols are deployed and configured. The scenario for this work has five spoke sites and one central hub to connect these sites. Ping request sent by one spoke site to other is represented by dotted line. Sample scenario for ping request from one spoke site to another can be as follows:

- Node 15 of site A sending a ping request to node 15 of site B.

- Node 3 sending a ping request to the core hub.

- Node 15 of site E sending the ping request to the node 15 of site D.

OSPF and EIGRP protocols are deployed on all routers in the network. The autonomous system number used for this network is 150. Each spoke site has multiple routers and switches. Multiple LANs exist in each spoke site which are connected with routers. Routers are linked to a central router through the switch. All spoke sites have same network structure. Eleven routers and one core switch are used at each spoke site which are directly linked to the central hub. Each spoke site consists of 10 LANs and multiple nodes. Fig. 3 shows a sample spoke site configured in this study.

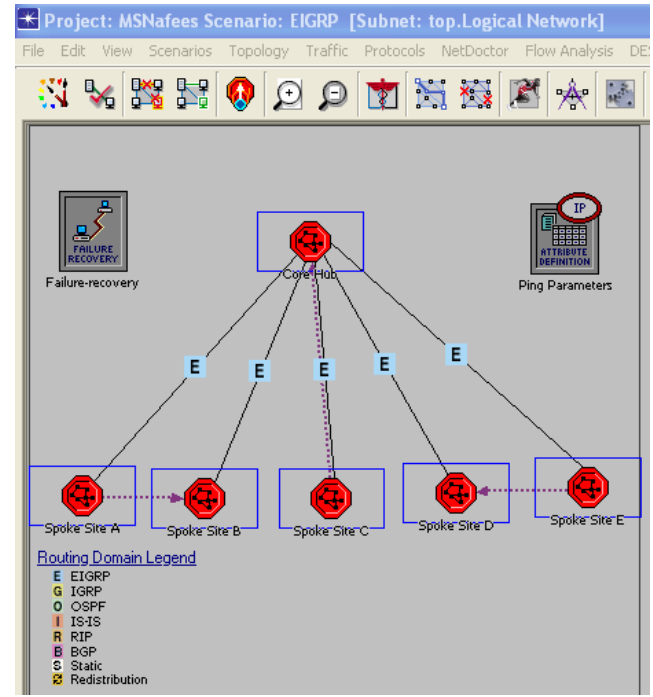

Fig. 1. EIGRP scenario.

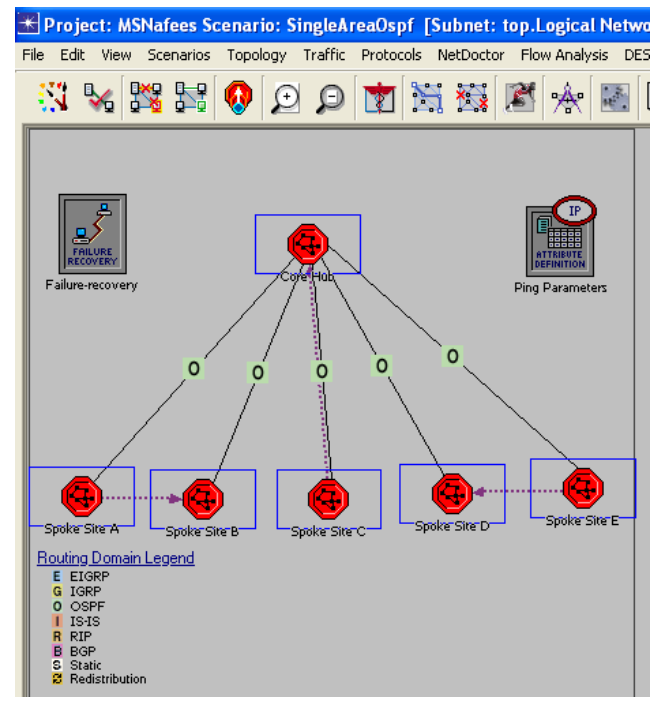

Fig. 2. OSPF scenario

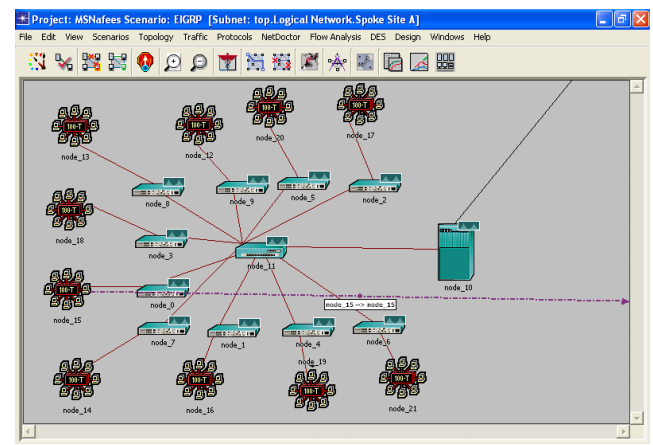

Fig. 3. Spoke Site A.

Central hub behaves like an intermediary among all spokes sites of network. Spoke sites are connected through this central hub (Fig. 4). Cisco 7500 series router is used as a central hub. 


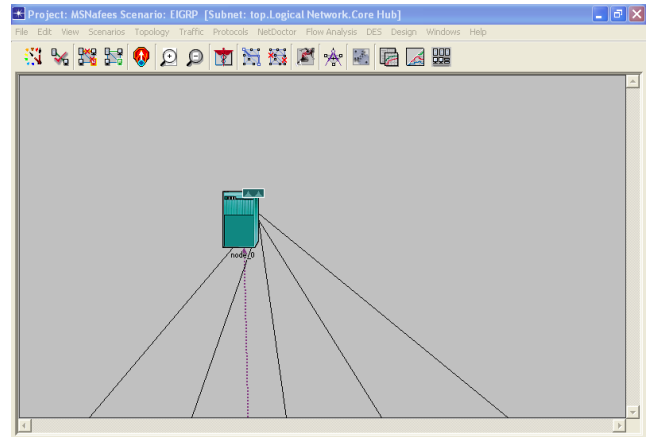

Fig. 4. Core Hub.

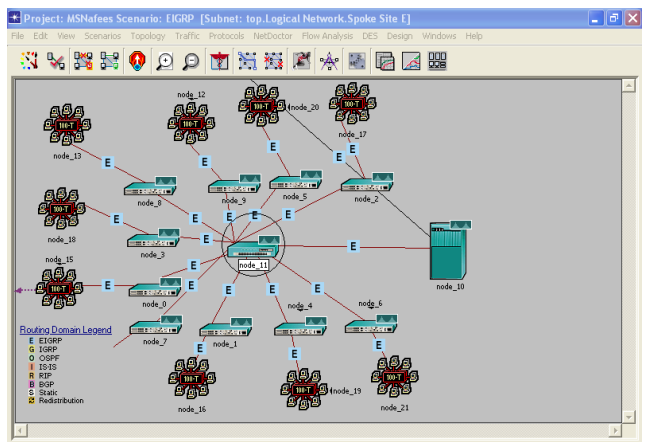

Fig. 5. Spoke Site E (EIGRP).

\section{A. EIGRP Scenario}

Fig. 5 shows that EIGRP is deployed on the routers of designed network. After the deployment of EIGRP the DES statistics are selected to examine the working of EIGRP.

\section{B. OSPF Scenario}

Fig. 6 shows a network that uses the OSPF in all of its routers. After the deployment of OSPF in this network the DES statistics are selected to evaluate the working of OSPF.

\section{Node Description}

Node models are created using Node Editor and then these node models are used for creating instances of node existing in network. A node is defined by connecting different modules

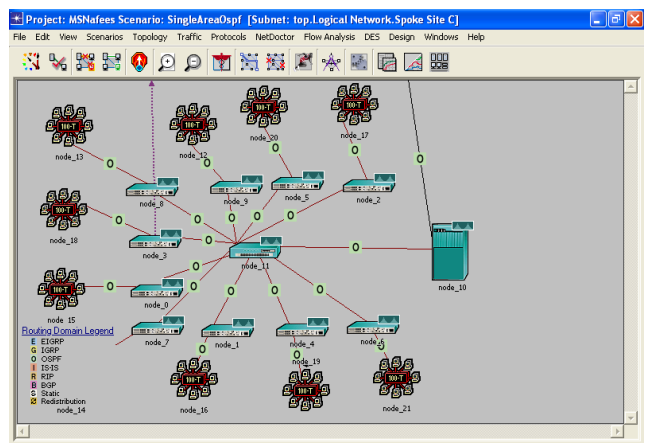

Fig. 6. Spoke Site C (OSPF).

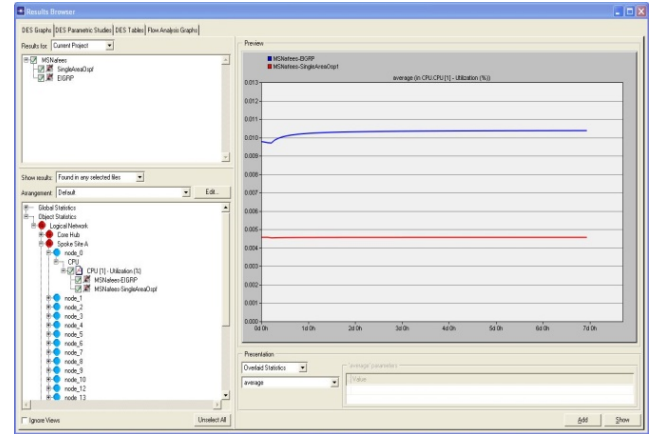

Fig. 7. Results browser for CPU utilization of Spoke Site A.

with packet streams and statistics. This connection among different modules allows packets and status to be exchanged. The modules in this connection serve for a specific purpose like producing packets, line up the produced packets, processing on packets and exchanging these packets. In our topology we use router 7609 as a core hub. The other nodes used in our topology are Cisco router 7513 and switch 3000.

\section{RESUlts AND Discussion}

The graphs in this section are the resultant of simulation. We compared the graphs of CPU Utilization for node_0 for each of the spoke sites. The resultant full window of CPU Utilization of node_0 of spoke site A is shown in Fig. 7. As shown in the figure, the CPU Utilization for both EIGRP and OSPF are checked in the window's left side. Then the right side shows the graph for both protocols. The result browsers for all the spoke sites are same as shown for spoke site A but the resultant graphs are different those are explained in this section.

The axis description for all the graphs is as follows:

- Time duration for CPU Utilization is represented on $\mathrm{X}$-axis.

- Percentage for CPU Utilization at some specific time is represented on Y-axis.

\section{A. CPU Utilization of Node_O in all Spoke Sites}

Fig. 8 shows graph for CPU Utilization of Node_0 in all of the Spoke Sites. As shown in the figure the EIGRP curve in all sub figures changes its behavior along $\mathrm{y}$-axis and then after some time the curve goes parallel along $\mathrm{x}$-axis. But on the other hand the OSPF curve goes parallel along $\mathrm{X}$-axis from the start of the simulation. As shown in the graph the behavior of OSPF curve is better than the EIGRP curve. This performance of OSPF is because of exchanging less routing updates than EIGRP. EIGRP needs the same information again and again so EIGRP wastes time and resources more than OSPF.

As shown in the graphs the behavior of the OSPF is better than the behavior of the EIGRP because the EIGRP is less intelligent than OSPF. The curve for EIGRP is illustrating the different behavior in each graph but the curve for OSPF is same in behavior. 


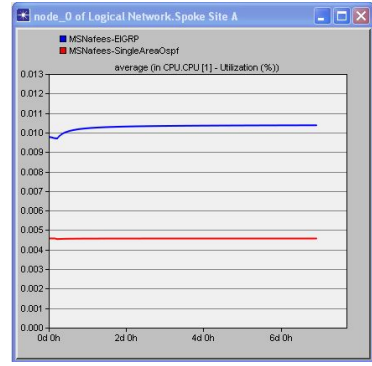

(a) Spoke Site A

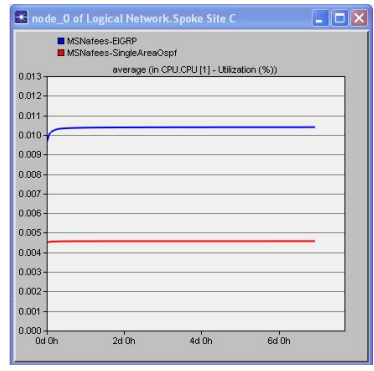

(c) Spoke Site C

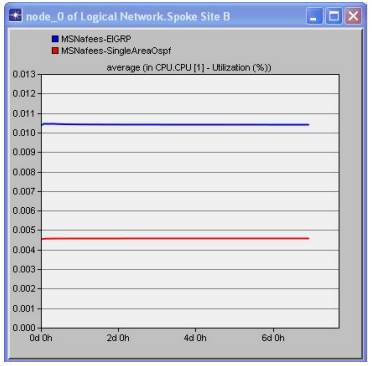

(b) Spoke Site B

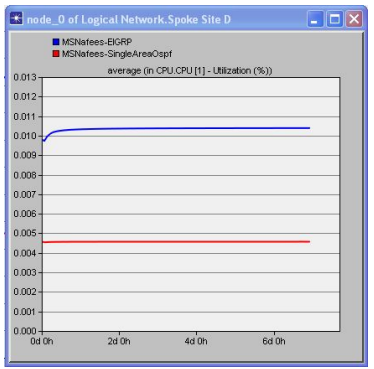

(d) Spoke Site D

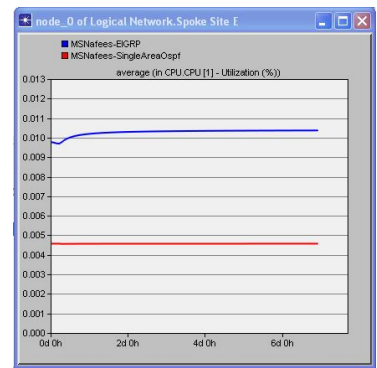

(e) Spoke Site E

Fig. 8. CPU utilization of Node_0 in Spoke Sites.

\section{CONClusion}

Amongst the several findings of our working the most important was about CPU Utilization. So, in this paper the investigating results showed that EIGRP deals with more routing information like additions, deletions and updates than the OSPF. As EIGRP requires updated information again and again and OSPF relies on previous information so the repeated steps in OSPF are reduced. The resultant investigation of our research describes that EIGRP acquires same information again and again so being less intellectual than OSPF it wastes time and resources like CPU.

\section{REFERENCES}

[1] D. Xu and L. Trajkovic, "Performance analysis of rip, eigrp, and ospf using opnet," 2011.

[2] J. Singh and D. R. Mahajan, "Simulation based comparative study of rip, ospf and eigrp," International Journal of Advanced Research in Computer Science and Software Engineering, vol. 3, no. 8, 2013.

[3] M. Ericsson, M. G. C. Resende, and P. M. Pardalos, "A genetic algorithm for the weight setting problem in ospf routing," Journal of combinatorial optimization, vol. 6, no. 3, pp. 299-333, 2002.

[4] H. Pun, "Convergence behavior of rip and ospf network protocols," $\mathrm{Ph} . D$. dissertation, Simon Fraser University, 2001.

[5] A. Frantsi and H. Venalainen, "Testing demands and routing protocols," IEEE Journal on Selected Areas in Communications, vol. 17, no. 8, pp. $1-5,2006$.

[6] S. Agarwal, C.-N. Chuah, S. Bhattacharyya, and C. Diot, "Impact of bgp dynamics on router cpu utilization," in International Workshop on Passive and Active Network Measurement. Springer, 2004, pp. 278288.

[7] A. Shah and W. Rana, "Performance analysis of rip and ospf network using opnet," ICSI, vol. 10, no. 6, pp. 256-265, 2013.

[8] N. Nazumudeen and C. Mahendran, "Performance analysis of dynamic routingprotocols using packet tracer," International Journal of Innovative Researchin Science, Engineering and Technology, vol. 3, 2014.

[9] A. Kudtarkar, R. Sonkusare, and D. Ambawade, "Performance analysis of routing protocols for real time application," International Journal of Advanced Research in Computer and Communication Engineering, vol. 3, no. 1, pp. 5072-5072, 2014.

[10] V. Vetriselvan, P. R. Patil, and M. Mahendran, "Survey on the rip, ospf, eigrp routing protocols," International Journal of Computer Science and Information Technologies, vol. 5, no. 2, pp. 1058-1065, 2014.

[11] H. N. Patel and R. Pandey, "Extensive reviews of ospf and eigrp routing protocols based on route summarization and route redistribution." Int. Journal of Engineering Research and Applications, vol. 4, no. 9, pp. 141-144, 2014.

[12] M. Masood, M. Abuhelala, and I. Glesk, "A comprehensive study of routing protocols performance with topological changes in standard networks," International Journal of Electronics, Electrical and Computational System (IJEECS), vol. 5, no. 8, pp. 31-40, 2016.

[13] E. Stankoska, N. Rendevski, and P. Mitrevski, "Simulation based comparative performance analysis of ospf and eigrp routing protocols," 2016 . 